\title{
FEMD Algorithm for Effective Segmentation of CT Lung Images
}

\author{
Z. Faizal Khan, Ph.D \\ Department of Computer and Network \\ Engineering, \\ College of Engineering, Shaqra University, \\ Al Dawadmi, Kingdom of Saudi Arabia
}

\author{
Syed Usama Quadri, Ph.D \\ Department of Computer and Network \\ Engineering, \\ College of Engineering, Shaqra University, \\ Al Dawadmi, Kingdom of Saudi Arabia
}

\begin{abstract}
Medical Image segmentation is the most important step in extracting information from medical images. Segmentation of pulmonary Chest Computed Tomography (CT) images is a precursor to most of the pulmonary image analysis schemes. The purpose of lung image segmentation is to separate the voxels corresponding to lung tissue from the anatomy of the surrounding. In this paper, an automated image segmentation method has been proposed inorder to segment the region of interest present in the CT Lung slices. The proposed approach utilizes Fuzzy logic with Earth Mover's Distance (FEMD) based refinement methods. The final segmented output is further refined by morphological based operators. The performance of the proposed method is compared with various segmentation methods such as Canny Sobel and Prewitt and we have obtained an average segmentation accuracy of $79.4091 \%$ for segmenting CT lung images.
\end{abstract}

\section{Keywords}

Computed Tomography, Fuzzy logic, Earth Mover's Distance, Segmentation.

\section{INTRODUCTION}

Image segmentation is important for extracting and isolating the important details of an image. Many methods have been proposed by early researchers for the process of segmenting images. This segmentation process partitions the entire image into different meaningful regions with homogeneous characteristics [1]. The process of Segmentation has been done in the past for quantifying the tissue volumes, computer integrated surgery, reconstruction of volume of medical image slices etc. CT lung images helps in evaluating the stages of unwanted portions which were grown inside the lung. This process is used for diagnosing various pulmonary diseases present in the lungs such as lung cancer, Tuberculosis, Pulmonary embolism etc. This paper presents three stages for performing lung segmentation.

Stage 1: The left and right lung potions of the CT image is segmented and refined using fuzzy logic with EMD.

Stage 2: Apply EMD again for refinement of the segmented regions.

Stage 3: Apply morphological based refinement operations inorder to obtain the refined and correctly segmented region.

Remaining sections of this paper is organized as follows. Section II presents an overview of existing CT lung image segmentation methods. Section III depicts the proposed FEMD methodology for segmenting CT lung images in detail. Section IV explains the experimental results, and Section V provides the conclusions about this work and suggests some possible future enhancements.

\section{RELATED WORKS}

Armato et al [3] proposed an accurate lung region segmentation procedure called a multi-level thresholding for identifying the regions of interest (ROI) lung nodule. 5\% $17 \%$ of the lung nodules are undetected by their approach. Segmentation of lung high resolution CT (HRCT) images using a pixel-based approach was suggested by Garnavi et al.[2] These authors have used global-threshold segmentation, mathematical morphology, edge detection, noise reduction, and geometrical computations to achieve the defined ROIs.

Xujiong Ye et al. [7] presented a CAD method for detecting both solid nodules and ground-glass opacity (GGO) nodules in the lung image. A fuzzy based thresholding approach is used to segment the lung region. Their method results in fast computation. A combination of iterative gray level thresholding and background-removal operator was proposed by Antonelli et al [11]. In their work, the background images was not eliminated well due to the presence of high noise.

Zheng et al. [9] proposed a method for segmenting the lung region by an adaptive threshold based on value distributions. Component analysis and gray-level thresholding are used to segment the lung volume. Arfan Jaffar et al [4] proposed a combination of spatial Fuzzy C-Means and morphological techniques for segmenting the lung region from CT lung images. They have divided their work into three main stages namely Background removal, Preprocessing, and Morphological based operations. Among them, a new histogram background removal operator has been used to remove the pixels around the lungs. Moreover, preprocessing is performed by smoothing and removing the noise present in the segmented image. Finally, morphological operations are used to separate the edges and to fill the small holes present in the segmented lungs.

From the works found in the literature, it has been observed that most of the existing works used. Thresholding, Fuzzy C means clustering, and Background removal operators. However in case of medical images, the accuracy provided by various stages of algorithms is not sufficient to make effective decision making. Therefore, it is necessary to propose a new and effective method to enhance the accuracy of segmentation.

\section{MATERIALS AND METHODS}

The FEMD system is based on fuzzy processing. In this paper, the term fuzzy logic is used to explicit the details of the image at various depths of information. Fuzzy rules are developed and used for determining the optimum segmentation of the lung from the background of a CT image.

We have taken gray scale images from LIDC Lung database as each pixel can be analyzed using the proposed FEMD method. The basic block diagram of the proposed system is given in Fig. 1. 


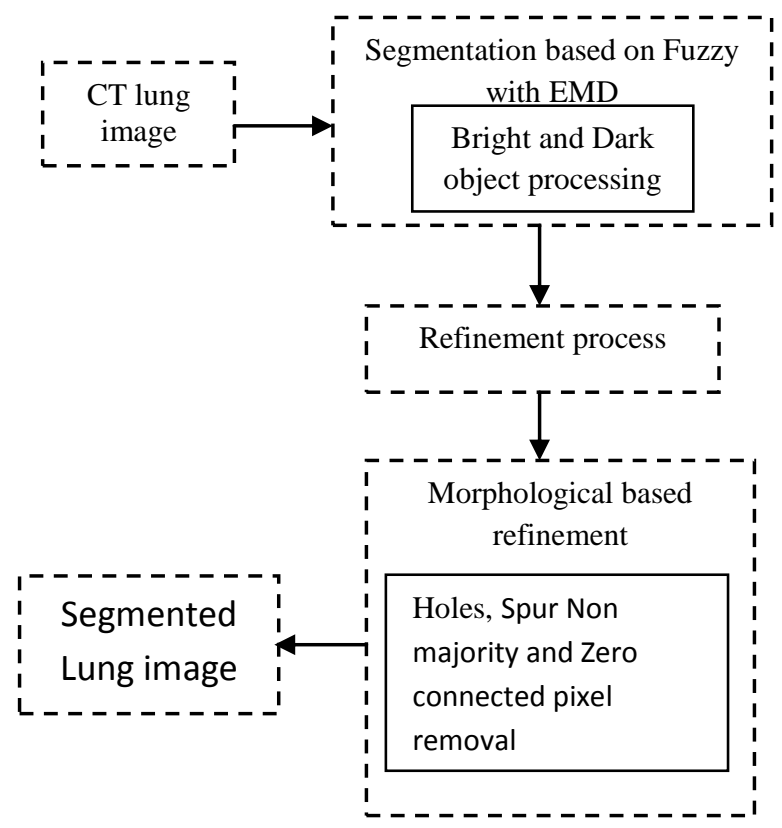

Figure.1. Block diagram of the proposed system.

Figure 1 is the prototype for the proposed system of pulmonary Lung region segmentation on CT Image which consists of three stages: (1) segmentation based on fuzzy with EMD (2) Refinement based on FEMD and (3) Morphological based refinement.

\section{SEGMENTATION BASED ON FUZZY LOGIC}

A fuzzy set is any set that allows its members to have different degree of membership, called membership function within the interval $[0,1]$. This can be mathematically expressed as $\mu A(x)$ which was assigned to each element on the set with in the same closed unit intervals. Let $X$ is the universal space and $\mathrm{x}$ is an element of the space $\mathrm{X}$. A fuzzy set an in space $\mathrm{X}$ is defined as

$A=\{\{x, A(X)\} \mid x \in X\}$.

Where $A(x)$ is the value of the function ' $A$ ' for element ' $x$ '. In this work, the ' $S$ ' function which denotes the set of brighter objects is used for modeling the membership function [5]. The Signum function based on fuzzy logic proposed in [12] can be defined as

$$
\begin{aligned}
& \mathrm{A}=\mu_{\mathrm{A}-\operatorname{sig}(\mathrm{x})}=\operatorname{sig}(x, a, b, c) \\
& \begin{cases}1 & b \leq x \leq c \\
0 & c \leq x \leq d \\
1 & x \geq d\end{cases}
\end{aligned}
$$

The parameters ' $a$ ' and 'c' controls the 'Sig' function. The value of ' $b$ ' can be computed as

$b=(1 / 2)(a+c)$ which is called cross over point. The mathematical function value of ' $b$ ' is $0.5(\mu A s(b)=0.5)$. The Sig (y) function, which is derived from the Sig (x) function which is used to represent the dark pixels is as follows

$$
\begin{aligned}
\mathrm{A} & =\mu_{\mathrm{A}-\operatorname{sig}(\mathrm{y})}=\operatorname{sig}(x, a, b) \\
& =\left\{\begin{array}{lll}
0 & x & \leq a \\
1 & a & <x \leq b
\end{array}\right.
\end{aligned}
$$

\section{REFINEMENT PROCESS}

Improving the quality of segmented output based on fuzzy methodology is carried in this stage. Histogram based analysis was done in order to improve the segmented result. The segmented output has been further refined by EMD [6] based refinement to reduce the issues in the existing methods [10]. For dark and white objects, the refinement process is carried using the following steps:

Step1: Compute the inside and outside histogram ' $\alpha$ ' and ' $\beta$ '.

Step2: Compute the inside and outside cumulative sum ' $\mathrm{I}_{c}$ ', ' $\mathrm{O}_{c}$ '.

$$
\begin{gathered}
\mathrm{I}_{c}\left(x_{i}\right)=\sum_{k=0}^{i} \alpha_{k} \\
i \rightarrow 0 \text { to } 255 \\
\mathrm{O}_{c}\left(x_{i}\right)=\sum_{k=0}^{i} \beta_{k}
\end{gathered}
$$

Step 3: For all black and white pixels $\mathrm{x}$ in the fuzzy region, compute the block histogram ' $\gamma$ '.

Step 4: For all black and white pixels, compute the foreground distance $\delta_{f}$

$$
\delta_{f}=\sum_{i=0}^{255} a b s\left\{\left(\sum_{k=0}^{i} \gamma_{k}\right)-\left(\sum_{k=0}^{i} \alpha_{k}\right)\right\}
$$

Where ' $\gamma_{k}$ ' and ' $\alpha_{k}$ ' are, respectively, the block histogram and inside histogram of white and dark objects.

The background distance ' $\delta_{b}$ ' is as follows:

$$
\delta_{b}=\sum_{i=0}^{255} a b s\left\{\left(\sum_{k=0}^{i} \gamma_{k}\right)-\left(\sum_{k=0}^{i} \beta_{k}\right)\right\}
$$

Step 5: The membership value presents the state of final marked image as ' 1 ' or ' 0 ' based on the below condition. 
Membership value $=\left\{\begin{array}{l}1 \text { if } \delta_{f}\left(x_{i}\right)<\delta_{b}\left(x_{i}\right) \\ 0 \text { else }\end{array}\right.$

\section{MORPHOLOGICAL BASED REFINEMENT}

We now use the morphological based refinement for further improving the quality of initial refined output, which contains the holes, spur, non-majority pixels and zero connective pixels. Predefined MATLAB morphological operators such as labelling was used to find the island slots within the lung regions and fill them up. The operation is as follows.

$p^{! !}=p^{!}+\operatorname{labeling}(\bar{p} !, v)$

where $\bar{p}$ ! denotes the set of island pixels that are not part of $p^{!}$. Labeling $(\bar{p} !, v)$ returns all components in $\bar{p}$ ! that contain at most $\mathrm{v}$ pixels $(\mathrm{v}=5000)$. An example of a complete segmented lung region after the labeling operation is shown in table 1 .

\section{RESULTS AND DISCUSSION}

In order to represent the performance of our proposed method, we have chosen $15 \mathrm{CT}$ images from LIDC Lung database. A ground truth image has been generated manually for each image. The ground truth image was combined with its corresponding original image, to segment the CT lung image. The performance of our proposed segmentation method has been compared with other conventional segmentation algorithms. The performance of our method over Lung image was carried out and the results are depicted in table 1 and the comparison between various conventional segmentation methodologies were shown in table 2. From the Table 2, it has been observed that the segmentation accuracy of our proposed method is greater in terms of accuracy for segmenting the CT lung images.
The segmentation accuracy $A_{s}$ is computed using the following equation to evaluate the quality of the segmentation results:

$$
\mathrm{A}_{\mathrm{s}}=\frac{\mathrm{S}_{\mathrm{I}}}{\mathrm{O}_{\mathrm{I}}} * 100
$$

$$
\mathrm{S}_{\mathrm{I}}=\left(\frac{(\text { 'Solidity'+' Area'+'Perimeter' })}{\text { Number of Objects }}\right)_{\text {Segmented Region }}
$$

and

$$
\mathrm{O}_{\mathrm{I}}=\left(\text { ' Solidity }^{\prime}+\text { 'Area'+' Perimeter' }\right)_{\text {Unsegmentel }} \text { Region }
$$

Where $\mathrm{S}_{\mathrm{I}}$ denotes the ratio between $\mathrm{O}_{\mathrm{I}}$ and the number of objects presented in the segmented region. $\mathrm{O}_{\mathrm{I}}$ denotes the total of solidity, Area and Perimeter of the pixels present in the unsegmented region.

Solidity is a scalar specifying the proportion of the pixels in the convex hull that are also in the region [13].

Area is the actual number of pixels in the region of interest. Convex Area is a Scalar that specifies the number of pixels in Convex Image. Convex Image is a Binary image (logical) that specifies the convex hull, with all pixels within the hull filled in. The image is the size of the bounding box of the region. This property is supported only for 2-D input label matrices. Perimeter is the distance around the boundary of the region. Region props compute the perimeter by calculating the distance between each adjoining pair of pixels around the border of the region.

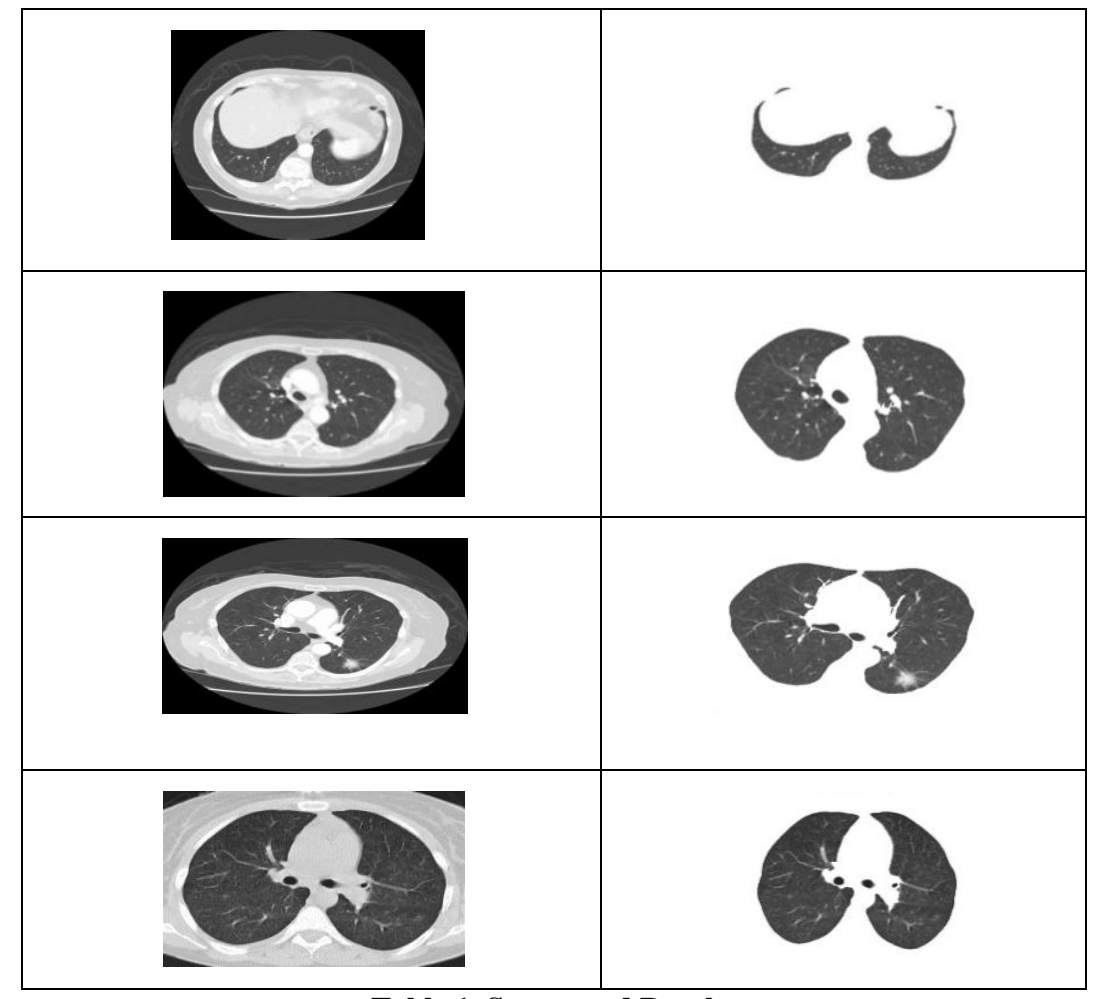

Table.1. Segmented Results 
Table 2. Segmentation accuracy of proposed method

\begin{tabular}{|l|c|c|c|c|c|}
\hline Algorithms & No of objects & 'Area' & 'Solidity' & 'Perimeter' & Segmentation \% \\
\hline Sobel & 57 & 43 & 0.253 & 96.5 & 5.0909 \\
\hline Prewitt & 40 & 40 & 0.292 & 93.4 & 4.8727 \\
\hline Robertz & 52 & 5 & 0.100 & 11.3 & 0.5964 \\
\hline Log & 40 & 80 & 0.102 & 197.0 & 10.0727 \\
\hline Zerocross & 40 & 80 & 0.102 & 197.0 & 10.0727 \\
\hline Canny & 33 & 115 & 0.150 & 194.0 & 11.2364 \\
\hline FEMD & 1 & 2490 & 0.6060 & 226.0 & 79.4091 \\
\hline
\end{tabular}

\section{CONCLUSIONS}

In this paper, we have proposed an automated Lung segmentation scheme based on fuzzy logic along with FEMD based refinement. The main advantage of this work is that it reduces false segmentation by increasing segmentation accuracy. Our method is useful in assisting for further diagnosis of Lung nodules in LDCT by reducing the mortality rate. Further works in this direction can be the application of a suitable classification algorithm for identification of Lung Nodules.

\section{REFERENCES}

[1] Wei Cao, Yixin Yan, Shengming Li, Unsupervised colortexture image segmentation based on a new clustering method, Journal of Next Generation Information Technology Vol. 1, Issue 2, August 2010, pp.784-787.

[2] Garnavi R, Baraani-Dastjerdi A, Abrishami Moghaddam H, 2005, A new segmentation method for lungHRCT images, Proceedings of the Digital Imaging Computing: Techniques and Applications, p.8. IEEE CS Press, Cairns Convention Centre, Brisbane, Australia, $<$ doi>10.1109/DICTA.2005.5.

[3] Armato S.G. and Sensakovic W.F., Automated Lung segmentation for thoracic CT, Acad. Radiol., Vol.11, Issue 9, 2004, pp.1011-1021.

[4] M. ArfanJaffar, Ayyaz Hussain, Anwar Majid Mirza," Fuzzy Entropy Based Optimization of Clusters for the Segmentation of Lungs in CT Scanned Images", Knowledge Information Systems, Vol. 24, pp. 91-111, 2010.

[5] Nuno Vieira Lopes, Pedro A., Mogadouro do Couto, and Humberto Bustince, Automatic histogram threshold using fuzzy measures, IEEE Transactions On Image Processing, Vol. 19, Issue 1, January 2010, pp.199-204.
[6] Amit Adam, Ron Kimmel and Ehud Rivlin, On scene segmentation and histograms-based curve evolution, IEEE Transactions on pattern analysis and machine intelligence, Vol. 31, Issue 9, September 2009, pp.17081714

[7] Xujiong Ye., Xinyu Lin, Jamshid Dehmeshki, Greg Slabaugh, and Gareth Beddoe, Shape-based computeraided detection of Lung nodules in thoracic CT Images, IEEE Transactions on Biomedical Engineering, Vol. 56, Issue 7, July 2009, pp.1810-1820.

[8] Zheng B., Leader J.K., Maitz G.S., Chapman B.E., Fuhrman C.R., Rogers R.M., Sciurba F.C., Perez A., Thompson P., Good W.F., and Gur D., A simple method for automated Lung segmentation in X-ray CT images, Proc. SPIE (Medical Imaging), Vol. 5032, 2003, pp.1455-1463.

[9] Faizal Khan, Z., and Kavitha, V., 2012, Pulmonary lung segmentation in Computer tomography using Fuzzy logic, European Journal of Scientific Research, Vol.81, No.3, pp.329-337.

[10] Antonelli M, Lazzerini B, Marcelloni F "Segmentation and Reconstruction of the Lung Volume in CT Images". 20th annual ACM symposium on applied computing, vol I. Santa Fe, New Mexico, pp. 255-259, March 2005.

[11] Faizal Khan, Z \& Kannan, "Intelligent Segmentation of Medical images using Fuzzy Bitplane Thresholding", "Measurement science and Review, Vol 14, No 2, pp-94101, 2014.

[12] Hoffman EA, McLennan G, "Assessment of the pulmonary structure-function relationship and clinical outcomes measures Quantitative volumetric CT of the lung”, AcadRadiol, Vol. 4, No. 11, pp. 758-776, 1997. 